\section{Gene sll0033 from Synechocystis 6803 Encodes a Carotene Isomerase Involved in the Biosynthesis of all- $E$ Lycopene}

Jürgen Breitenbach ${ }^{\mathrm{a}}$, Agustin Vioque ${ }^{\mathrm{b}}$ and Gerhard Sandmann ${ }^{\mathrm{a}, *}$

${ }^{a}$ Biosynthesis Group, Botanical Institute, J. W. Goethe Universität, P. O. Box 111932, D-60054 Frankfurt, Germany. E-mail: sandmann@em.uni-frankfurt.de

b Instituto de Biochímica Vegetal y Fotosíntesis, Universidad de Sevilla-CSIC, Americo Vespucio s/n, 41092, Spain

* Author for correspondence and reprint requests

Z. Naturforsch. 56 c, 915-917 (2001); received August 7, 2001

Carotene Isomerase, Gene sll0033, all-E Lycopene

The function of gene sll0033 from Synechocystis 6803 which is homologous to the bacterial crtI-type phytoene desaturase genes was elucidated as a novel carotene isomerase. Escherichia coli transformed with all genes necessary for the formation of $\xi$-carotene and expressing a $\zeta$-carotene desaturase synthesized the positional isomer prolycopene $\left(7,9,7^{\prime}, 9^{\prime} Z\right.$ lycopene) which cannot be cyclized in the subsequent reactions to a- and $\beta$-carotene. Upon cotransformation with sll0033, the formation of all- $E$ lycopene is mediated instead.

In cyanobacteria and plants, $\alpha$-carotene, $\beta$-carotene and their derivatives dominate as all- $E$ isomers. However, among the carotenogenic mutants, prolycopene $\left(7,9,7^{\prime}, 9^{\prime} \mathrm{Z}\right.$ lycopene) accumulating Scenedesmus obliquus (Ernst and Sandmann, 1988) and tangerine tomato fruit (Clough and Pattenden, 1983) can be found. Enzymological studies with plastids of Narcissus pseudonarcisssus indicated that the reaction product of the plant-type $\zeta$-carotene desaturase (encoded by crt $Q b$ in cyanobacteria or $z d s$ in plants) is poly-cis prolycopene (Beyer et al., 1991). Due to its steric configuration, this isomer cannot be cyclized to ionone end groups. Therefore in the carotenogenic pathway of cyanobacteria and eukaryotes with a similar $\zeta$-carotene desaturase, a specific process may prevent the accumulation of prolycopene or convert it mainly to the all- $E$ form which then can be further cyclized. This isomerization reaction may be catalyzed by a specific enzyme. It is the objective of this study to identify and demonstrate the existence of this carotene isomerase.
Complementation studies in Escherichia coli with different carotene background identified the function of the genes in the Synechocystis 6803 genome (Kaneko et al., 1996) related to pds- and crtI-type carotene desaturases (Fernández-González et al., 1997; Breitenbach et al., 1998) with the exception of sll0033 belonging to the latter type. The role of the corresponding protein in carotenogenesis remained open. Since CrtI and structurally-related CrtQa enzymes both produce all- $E$ lycopene as their reaction products, the participation of sll0033 in the formation all- $E$ lycopene was investigated. For this purpose, E. coli was cotransformed with pACCRT-EBP which mediated the formation of $\xi$-carotene (Linden et al., 1993), pBBR1MCS2zds carrying the $\zeta$-carotene desaturase cDNA from Capsicum annuum (Breitenbach et al., 1999) and with plasmid pTrc-0033 containing gene sll0033 (Breitenbach et al., 1998). In the control transformant, the latter plasmid was replaced by the cloning vector pTrc99A. Growth in the presence of ampicillin, kanamycin, chlorampheni-

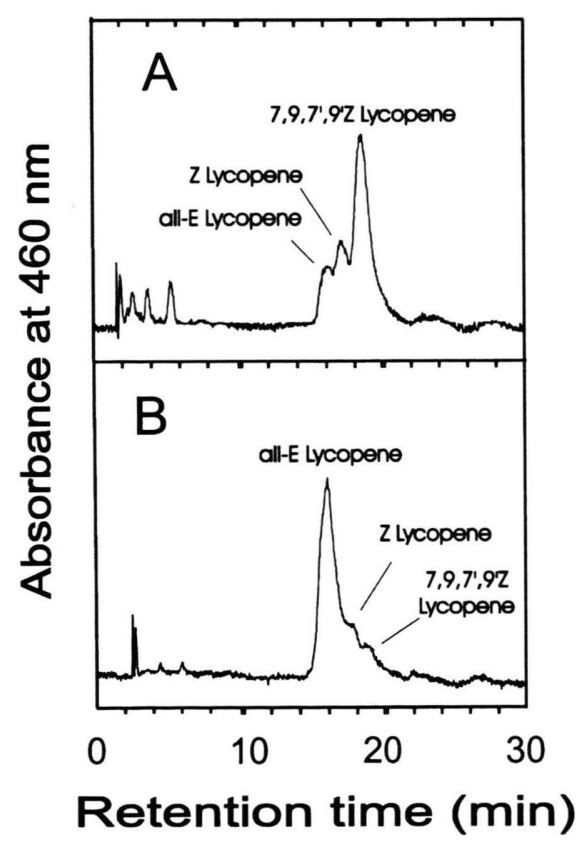

Fig. 1. HPLC separation of carotenoids from E. coli/ pACCRT-EBP/pBBR1MCS2zds/pTrc99A (A) and $E$. coli/pACCRT-EBP/pBBR1MCS2zds/pTrc-0033 (B). Separation was carried out on a Nucleosil $\mathrm{C}_{18} 3 \mu$ column with acetonitrile/methanol/2-propanol (85:10:5). 
col and isopropyl $\beta$-D-thiogalactopyranoside was for $20 \mathrm{~h}$ at $26^{\circ} \mathrm{C}$. Details of the cultivation conditions, carotenoid extraction and HPLC analysis of the carotenoids are given in former publications (Breitenbach et al., 1998 and 1999). Identification of the lycopene isomers was performed as recently described (Breitenbach et al., 2001).

Fig. 1 shows the HPLC separation of the carotenoids from $E$. coli/pACCRT-EBP/pBBR1MCS2zds/pTrc-99A without sll0033 as a control (A) and the transformant expressing sll0033 additionally (B). $\zeta$-Carotene desaturase alone mediates the formation of mainly prolycopene $\left(7,9,7^{\prime}, 9^{\prime} Z\right)$ and small amounts of all- $E$ lycopene (Fig. 1A). The latter may result from photoisomerization to which prolycopene is very susceptible. The $Z$-lycopene peak running between prolycopene and all- $E$ lycopene consists mainly of $7 \mathrm{Z}$ and other lycopene isomers. The quantitative distribution of the lycopene isomers is $\mathrm{x} 15 \%$ all- $E, 19 \% Z$ and $66 \%$ prolycopene. Dominance of prolycopene as the reaction product was also observed for the z-carotene desaturase CrtQb from Synechocystis (Breitenbach et al., 2001). In the presence of pTrc-0033 (Fig. 1B), all- $E$ is the major product of $\xi$-carotene desaturation representing $79 \%$ of the lycopene isomers to- gether with $11 \%$ all- $E$ and $10 \%$ prolycopene. From this result it can be concluded that sllo033 encodes a protein which either isomerizes prolycopene to the all- $E$ form or shifts the isomer composition to all- $E$ during $\xi$-carotene desaturation. In vitro studies with the isolated S1l0033 should tell whether this protein is acting either simultaneously with $\zeta$-carotene desaturase to prevent the formation of prolycopene instead of all- $E$ lycopene or sequentially as an independent prolycopene isomerase converting this poly-cis isomer to all- $E$ lycopene.

Formation of all- $E$ lycopene in the carotenogenic pathway can be catalyzed by combination of different gene products or by CrtI alone (Fig. 2). The 4-step desaturation by CrtI is the ancient bacterial/archebacterial desaturation reaction. During evolution, this enzyme was replaced by a newly acquired 2-step phytoene desaturase Pds at the stage of cyanobacteria. As a consequence, a second $\mathrm{z}$-carotene desaturase is required to catalyze the two remaining desaturation steps. In Anabaena 7120 , this function is exerted by CrtQa which evolved from CrtI and like CrtI produces all- $E$ lycopene. In other cyanobacteria like Synechococcus and Synechocystis, $\zeta$-carotene desaturation is cata-

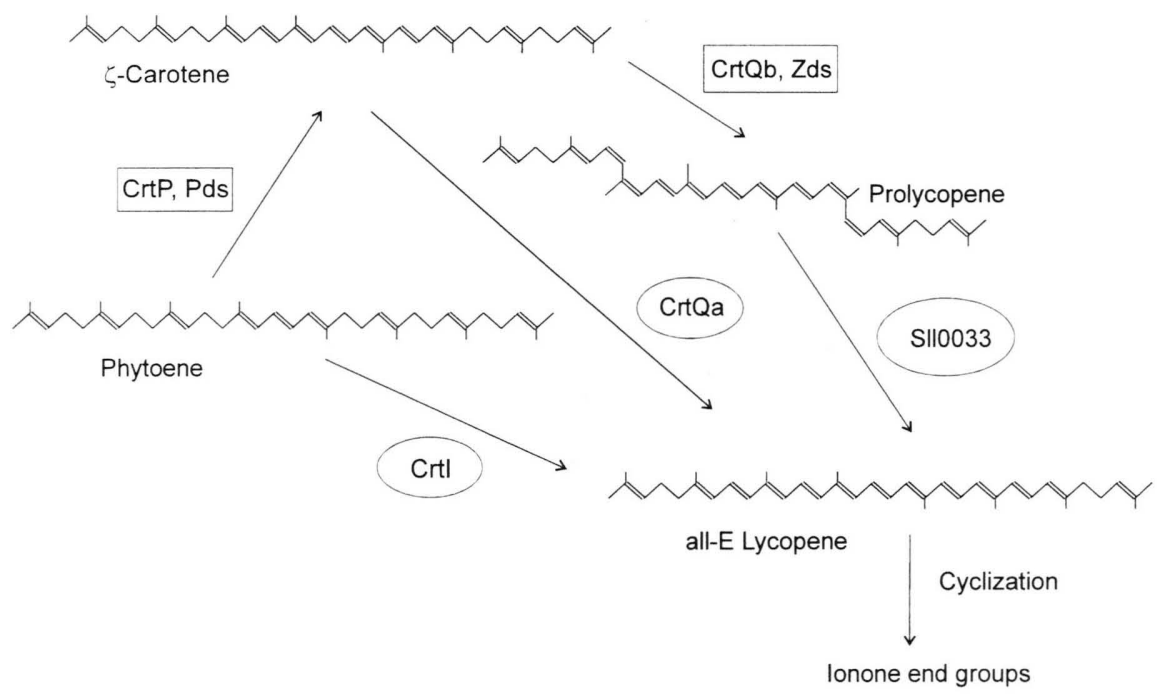

Fig. 2. Reaction sequences from phytoene to all- $E$ lycopene involving different gene products. CrtI, 4-step phytoene desaturase from bacteria or archebacteria; CrtP and Pds, 2-step phytoene desaturases from cyanobacteria and plants; CrtQb and Zds, $\zeta$-carotene desaturase from cyanobacterial and plants; CrtQa, $\zeta$-carotene desaturase from Anabaena 7120; Sll0033 carotene isomerase from Synechocystis 6803. Boxes and cicles indicate the structual relationship to the pds- or crtI-type of carotene desaturases, respectively. 
lyzed by $\mathrm{CrtQb}$ (formerly named CrtQ-2) from which the plant $\xi$-carotene desaturase evolved (Sandmann and Vioque, 1999).

Obviously, in the course of evolution of carotenogenesis from bacteria via cyanobacteria to plants, the simple situation of one enzyme for the entire reaction sequence from phytoene to all- $E$ lycopene changed to a more complex process involving three individual enzymes, a novel phytoene and $\xi$ carotene desaturase as well as a carotene isomerase which is phylogenetically related to CrtI.

Beyer P., Kröncke U. and Nievelstein V. (1991), On the mechanism of the lycopene isomerase/cyclase reaction in Narcissus pseudonarcissus L. chromoplasts. J. Biol. Chem. 266, 17072-17078.

Breitenbach J., Fernandez-Gonzales B., Vioque A. and Sandmann G. (1998), A higher- plant type $\zeta$-carotene desaturase in the cyanobacterium Synechocystis PCC6803. Plant Molec. Biol. 36, 725-732.

Breitenbach J., Kuntz M., Takaichi S. and Sandmann G. (1999), Catalytic properties of an expressed and purified higher plant type $\zeta$-carotene desaturase from Capsicum annuum. Eur. J. Biochem. 265, 376-383.

Breitenbach J., Braun G., Steiger S. and Sandmann G. (2001), Chromatographic performance on a $\mathrm{C}_{30^{-}}$ bonded stationary phase of mono hydroxycarotenoids with variable chain length or degree of desaturation and of lycopene isomers synthesized by different carotene desaturases. J. Chromatogr. A, in press.

Clough J. M. and Pattenden G. (1983), Stereochemical assignment of prolycopene and other poly-cis isomeric carotenoids in fruits of the tangerine tomato Lycopersicum esculentum var. "Tangella". J. Chem. Soc. Perkin Trans I, 3011-3018.
Only the CrtI-type enzymes seem to have the property to catalyze the formation of all- $E$ lycopene, the most important isomer for the formation of cyclic carotenoids. In addition to the isomerase gene sll0033 from Synechocystis, a highly similar sequence AAF631149 (Blast score of 592, E-value of $10^{-168}$ ) is present in the data base of the complete Arabidopsis thaliana genome sequence. This gene is the best candidate for a corresponding plant carotene isomerase gene.

Ernst S. and Sandmann G. (1988), Poly-cis carotene pathway in the Scenedesmus mutant C-6D. Arch. Microbiol. 150, 590-594.

Kaneko T., Sato S., Kotani H., Tanaka A., Asamizu E., Nakamura Y., Miyajima N., Hirosawa M., Sugiura M., Sasamoto S., Kimura T., Hosouchi T., Matsuno A., Muraki A., Nakazaki N., Naruo K., Okumura S., Shimpo S., Takeuchi C., Wada T., Watanabe A., Yamada M., Yasuda M. and Tabata S. (1996), Sequence analysis of the genome of the unicellular cyanobacterium Synechocystis sp. strain PCC6803. II. Sequence determination of the entire genome and assignment of potential protein-coding regions. J. DNA Res. 3, $109-136$.

Linden H., Vioque A. and Sandmann G. (1993), Isolation of a carotenoid biosynthesis gene coding for a zeta-carotene desaturase from Anabaena PCC7120 by heterologous complementation. FEMS Microbiol. Lett. 106, 99-104.

Sandmann G. and Vioque A. (1999), Phylogeny of carotenogenic genes and enzymes in cyanobacteria. In: The Phototrophic Prokaryotes (Pescheck G A., Löffelhardt W., Schmetterer G., eds.). Kluwer Academic, New York, pp. 799-803. 